\title{
Prinzipien der botanischen Nomenklatur in der Renaissance
}

Philippe Selosse

Université Lumière, Lyon 2

The aim of this paper is to bring to light the pattern underlying botanical nomenclature in the Renaissance. The analysis shows that this nomenclature was conceived within the framework of the Renaissance episteme, i.e. within a logical and religious framework and that it is characterized by a threefold purpose: the appellations must give an essential definition of plants, reflect the classification of the botanists, and be easy to remember, that is, they must be short. Consequently, the nomenclature is structured by various principles: philosophical (logical division), linguistic (anaphora, polysemy) and cognitive (typicality). Finally, the analysis leads to a definition of the concept of 'nomenclature' in the Renaissance.

\section{Einleitung}

Die moderne botanische Nomenklatur nach Linné, sowie die Taxonomie, die sie widerspiegelt, benutzt fixe Kategorien (Art, Gattung, Familie, Ordnung), die ihre eigenen Definitionen, Kriterien und ihre eigene hierarchische Stellung haben. Im Einzelnen besteht der Name jeder Pflanze aus einem den Namen einer Gattung und den Namen einer Art umfassenden Binom. Ein Baldrian zum Beispiel wird durch das nach der modernen Linné'schen Nomenklatur zusammengesetzte Binom (1) bezeichnet:

(1) Valeriana officinalis

Offizinaler Baldrian

wobei das Epitheton officinalis die Art unterscheidet, während der Name Valeriana ausdrückt, dass die Pflanze der Gattung der Baldriane angehört. Dieses Strukturmodell dient im Allgemeinen als Grundlage zur Erklärung der Renaissancenomenklatur, unabhängig vom Aufbau der Pflanzennamen. Im Falle einer Benennung ${ }^{1}$ der Renaissance wie z.B. (2)

Valeriana caerulea.

Blauer Baldrian.

finden die modernen Interpreten (Möbius 1937: 38; Hünemörder 1983; Pajaud 1989: 46) ein Linné'sches Binom wieder, das sich aus einer monolexikalischen Erstkonstituente, einem Gattungsnamen (Valeriana), und aus einer monolexikalischen Zweitkonstituente, einem spezifischen Epitheton (caerulea), zusammensetzt. Aber diese Interpreten erkennen ebenfalls diese binomische oder binarische Struktur der Linné'schen Nomenklatur in der Benennung (3): 
Valeriana palustris media parum laciniata.

Wenig gelappter mittelgroßer sumpfiger Baldrian.

in der ein Gattungsname (Valeriana) durch ein spezifisches Epitheton wie palustris media parum laciniata ergänzt wird. In diesem Fall erscheint die spezifische Beifügung nicht als ein adjektivisches Monom, sondern als ein Polynom: wegen dieser polynomischen Form wird die in den Beispielen (2)-(3) illustrierte Renaissancenomenklatur von den modernen Botanikern und Historikern (siehe z.B. Atran 1987) als variabel, heterogen, ohne Regeln, in einem Wort: volkstümlich - bestenfalls vorwissenschaftlich betrachtet.

Im Gegensatz zu dieser modernen Interpretation der Botaniker und Historiker geht es in diesem Artikel darum, die wichtigsten syntaktischen und semantischen Prinzipien aufzudecken, die die Renaissancenomenklatur strukturieren. Anstatt von den in der modernen Zeit entstandenen a priori Begriffen (Art und Gattung, binomische Nomenklatur, Taxonomie) auszugehen, werde ich mit einer linguistischen Untersuchung der Pflanzenbenennungen der Renaissance beginnen. Gleichzeitig werde ich die ermittelte Struktur auf kognitiver Ebene untersuchen um zu zeigen, auf welche Weise die linguistische Nomenklaturstruktur die begriffliche Struktur der Botanik der Renaissance widerspiegelt. Da die Nomenklatur des sechzehnten Jahrhunderts zu einer anderen Episteme ${ }^{2}$ als die Linné'sche Nomenklatur des zwanzigsten Jahrhunderts gehört, werde ich oft auf die Charakteristiken der Episteme der Renaissance zurückgreifen.

In diesem Artikel beschränkt sich die Untersuchung der botanischen Renaissancenomenklatur auf die Nomenklatur eines einzigen Autors, nämlich des Schweizer Botanikers Caspar Bauhin (1560-1624). Ich habe diese Nomenklatur als exemplarisch gewählt, weil sie zu einem Werk gehört, das alle für die Renaissance charakteristischen botanischen Werke (1530-1620) zusammenfasst, und das als Synthese von den späteren Botanikern lange Zeit als klassisches Nachschlagewerk betrachtet worden ist. Dieses Werk ist jedoch eine unvollendete Synthese: Es besteht nur aus immer vollständigeren Entwürfen des endgültigen Werkes, d.h. Theatrum Botanicum oder Historia Universalis Plantarum. Von der ersten (Phytopinax, 1596) bis zur letzten Schrift (Pinax Theatri Botanici, 1623) erscheint dieses Werk als ein umfassendes Verzeichnis von Namen, denen weder eine Beschreibung noch ein Holzschnitt oder eine Erklärung zur Nomenklatur- oder zur Klassifizierungsmethode beiliegt (s. Abbildung Nr. 1). Das erklärte Ziel ist keine Nomenklatur, sondern die "Aufzählung aller Pflanzen" und die Anfertigung eines "Index" aller Namen oder Synonyme, die die Botaniker von der Antike bis zur Renaissance einer Pflanze gegeben haben. Auch wenn die Nomenklatur Bauhins also nirgendwo in seinen Schriften erklärt wird, ist sie gerade deshalb doch interessant, weil sie über einen Zeitraum von vierzig Jahren entwickelt wurde: die Manuskripte, die Etiketten des Herbars (die ersten stammen aus dem Jahr 1578) und die ersten Druckwerke zeigen also unterschiedliche Versionen der Nomenklatur, durch deren diachronische und 
philologische Untersuchung sich die Prinzipien der linguistischen Struktur aufdecken lassen.

Das allerletzte Werk, der Pinax Theatri Botanici von 1623, enthält nahezu 5800 Pflanzen, die über zwölf Bücher verteilt sind: Die ersten acht Bücher sind die acht beträchtlich erweiterten Bücher des Phytopinax von 1596, zu denen vier andere Bücher hinzukommen; jedes Buch gliedert sich wiederum in sechs Sektionen und jede Sektion in unnummerierte Kapitel, die einen Namen in Großbuchstaben als Überschrift haben. Innerhalb der Kapitel (s. Abbildung Nr. 1) finden sich in Kleinbuchstaben gedruckte, römisch nummerierte Pflanzen, deren Nomenklatur Bauhin eigen ist. Der Nomenklatur Bauhins folgt eine sehr ausführliche bis zu 40 Namen umfassende Synonymie mit den Abkürzungen der Namen der Botaniker, nach denen die Pflanzen benannt wurden. Zur nachfolgenden Strukturuntersuchung der Renaissancenomenklatur, entnehme ich alle Beispiele den Kapiteln VALERIANA und NARDUS aus Buch IV, Sektion 6, sowohl im Phytopinax als auch im Pinax Theatri Botanici (s. Abbildung Nr. 1).

\section{Die logische Teilung, ein erstes strukturelles Prinzip}

Der Phytopinax von 1596 zeigt den frühesten Zustand der Nomenklatur, im Fall der Valeriana, zwölf in Kleinbuchstaben gedruckte, römisch nummerierte Pflanzen, eine nach der anderen:

(4) I. Valeriana hortensis. Garten-Baldrian.

(5) II. Valeriana sylvestris major. Grösserer wilder Baldrian.

(6) III. Valeriana sylvestris minor. Kleinerer wilder Baldrian.

(7) IV. Valeriana montana prima. Erster Berg-Baldrian.

(8) V. Valeriana montana bellidis foliis. Berg-Baldrian mit Maßliebchenblättern.

(9) VI. Valeriana montana altera. Anderer Berg-Baldrian.

(10) VII. Valeriana montana Alsines folio. Berg-Baldrian mit Miereblättern.

(11) VIII. Valeriana peregrina purpurea. Purpurner fremder Baldrian.

(12) IX. Valeriana montana inodora. Geruchloser Berg-Baldrian.

(13) X. Valeriana peregrina caerulea. Blauer fremder Baldrian.

(14) XI. Valeriana campestris inodora major. Grösserer geruchloser Feld-Baldrian. 


\author{
XII. Valeriana campestris inodora laciniata. \\ Gelappter geruchloser Feld-Baldrian.
}

Auf linguistischer Ebene haben alle aufgeführten polynomischen Benennungen einen Namen im Nominativ gemeinsam, d.h. Valeriana. Darüber hinaus sind beim Vergleich einiger dieser Benennungen gemeinsame Nominalgruppen zu erkennen: Valeriana sylvestris in den Benennungen (5)-(6), Valeriana montana in (7)-(10), Valeriana peregrina in (11) und (13), Valeriana campestris in (14)-(15). $\mathrm{Zu}$ diesen vier Syntagmen kommt das Syntagma Valeriana hortensis hinzu, da dieses Syntagma mit den anderen strukturell vergleichbar ist, sowohl auf syntaktischer Ebene (Substantiv + Adjektiv) als auch auf semantischer Ebene: das Epitheton hortensis bezeichnet einen Standort wie die anderen Adjektive sylvestris, montana, peregrina, campestris. Da stellt sich die Frage, ob die Zusammensetzung dieser Syntagmen einen Sinn ergibt, anders ausgedrückt, ob sie auf einen Begriff verweist, und wenn ja, auf welchen.

\title{
2.1. Die verschiedenen Gattungsstufen
}

Eine terminologische Besonderheit kann uns darüber Aufschluss geben. Der Index des Pinax verweist auf den Baldrian und seine Arten; aber die kurze, kursiv gedruckte Einleitung Bauhins zum Baldrian nennt verschiedene Gattungen von Baldrian. Die Austauschbarkeit der Termini, die man in allen Schriften von Bauhin feststellen kann, birgt jedoch keinen Widerspruch: Sie bedeutet einfach, dass Bauhin die Termini Gattung und Art entsprechend der "logischen Teilung" (Porphyrus 1998: 6) benutzt. In der Renaissance wird der Universitätsunterricht von der neoaristotelischen Logik beherrscht: das Wissen wird dichotomisch in Form von Bäumen oder Klammern, die von den neoaristotelischen Porphyrschen Bäumen stammen, dargestellt (Freedman 1993) und das definierende Verfahren, das zur Essenz der Gegenstände führt (Aristoteles 1991, I: 420; Aristoteles 1997: 10), wird "logische Teilung" genannt. In diesem Modell der logischen Teilung finden die vorher unterschiedenen Nominalgruppen einen Sinn.

Aufgrund ihrer Teilbarkeit, ist VALERIANA eine Gattung, auch "höchste Gattung" (genus supremum) genannt, die mehrere Arten umfasst: Valeriana hortensis, Valeriana sylvestris, Valeriana montana, Valeriana peregrina und Valeriana campestris. Doch da die vier letzten Arten ihrerseits teilbar sind, funktionieren diese als Gattungen, auch "nächsthöhere Gattungen" (genus proximum) oder "Zwischengattungen" (genus intermedium) genannt, die wiederum mehrere Arten wie (5)-(15) umfassen. Da diese letzten Arten nicht weiter unterteilt werden können, werden sie als "äußerste Arten" (ultima species) bezeichnet. Die Abbildung Nr. 2 fasst diese Erklärungen in Gestalt eines Porphyrschen Baums zusammen, so wie ich ihn rekonstruiert habe. Ein Porphyrscher Baum zeigt das Netz der Beziehungen 
zwischen den Pflanzen der Schöpfung, das die Botaniker der Renaissance ans Licht zu bringen versuchen.

\subsection{Die verschiedenen Gattungsnamen}

Die botanische Nomenklatur enthält also gemäß der betrachteten Teilungsstufe mehrere Gattungsnamen. In der ersten Teilung, die zum Beispiel zu Valeriana sylvestris führt, ist Valeriana der Gattungsname und sylvestris die definierende Differenz der Art. Aber in der zweiten Teilung, die zum Beispiel zu (5) Valeriana sylvestris major führt, bildet das Syntagma Valeriana sylvestris den Gattungsnamen, während das Adjektiv major die wesentliche Differenz der Art ist ${ }^{3}$.

Was die linguistische Erklärung und die epistemische Untersuchung also klarstellen, ist die Tatsache, dass zwischen dem in Großbuchstaben genannten Kapitel (VALERIANA) und den in Kleinbuchstaben gedruckten äußersten Arten (z.B. Valeriana sylvestris major) Zwischengattungen (z.B. Valeriana sylvestris) bestehen, obwohl die durchgehende römische Nummerierung (s. Abbildung Nr. 1) die Tendenz hat, diesen Bestand zu verschleiern.

Dreißig Jahre später, im Pinax von 1623, ist im Fall der Valeriana die logische Teilung immer noch das wichtigste Strukturprinzip ${ }^{4}$, obwohl neue Erkenntnisse über die Orte, an denen die Pflanzen vorkommen, einige Änderungen in Bezug auf die Zwischengattungen herbeigeführt haben. Pflanzen, die nicht mehr als wild (sylvestris) im Allgemeinen, d.h. an mehreren Orten wachsend, sondern als wild im spezifischen Sinne, d.h. an einem spezifischen Ort wachsend - im vorliegenden Fall Sümpfen (paludes) - betrachtet werden, bilden nun eine neue Zwischengattung: Valeriana palustris. Die Unterscheidung zwischen alpinen Orten (alpinus) und gebirgigen Orten (montanus) hat zum Ersatz der Gattung Valeriana montana durch die gemischte Gattung Valeriana alpina-montana geführt. Darüber hinaus hat auch die Entdeckung neuer Pflanzen in der Natur zu zwei neuen Zwischengattungen, Valeriana rubra und Valerianella (s. Abbildung Nr. 3) geführt. Der Pinax zeichnet sich durch eine typographische Neuheit aus, die es erlaubt, dass man sich aufgrund der Benennungen der Existenz der verschiedenen Gattungsstufen bewusst wird (s. Abbildung Nr. 4): die Benennung der höchsten Gattung erscheint in Großbuchstaben (Titel von Kapiteln); die der Zwischengattungen der zweiten Stufe in kleineren Großbuchstaben; die der Zwischengattungen der dritten Stufe in kursiven Kleinbuchstaben; die der äußersten Arten werden in römischen Kleinbuchstaben gedruckt. Doch diese neuartige Typographie fand keine systematische Verwendung. Der Grund dürfte wohl sein, dass die typographische Praxis in der Renaissance sehr wechselnd ist, weil die Buchdrucker ihre Bücher von Personen jedes Bildungsstandes verlegen lassen. Die Typographen, die manchmal nur auf ihre Ausbildung in der Werkstatt (so z.B. die Humanisten, die in den Druckereien arbeiten) zurückgreifen können, manchmal ungebil- 
det sind (z.B. der lateinischen Sprache nicht mächtig) und über keine Fachkenntnisse, wie z.B. in der Botanik, verfügen, machen oft Fehler oder wechseln die Drucktypen innerhalb eines Werkes. Demnach ist es durchaus möglich, dass im Pinax die Großbuchstaben, die kleineren Großbuchstaben oder die Kursivschrift einer Pflanzenbenennung sowohl eine Zwischengat-tung der zweiten Stufe als auch eine äußerste Art bezeichnen. Die Abbildung Nr. 1 illustriert dieses Problem: die Großbuchstaben von NARDUS bezeichnen eine höchste Gattung, während die Großbuchstaben von NARDUS MONTANA auf eine Zwischengattung der zweiten Stufe hinweisen. Was also bedeutend ist, ist vor allem die typographische Veränderung innerhalb eines Kapitels: wenn man in einem einzigen Kapitel eine Benennung in Großbuchstaben, dann andere Benennungen in kleinen Großbuchstaben oder in Kursivschrift, und zuletzt andere nummerierte Benennungen in Kleinbuchstaben findet, zeugt das von der Begriffsbildung und von der Bezeichnung der verschiedenen Stufen einer logischen Teilung.

\subsection{Die wesentliche Pflanzendefinition}

Die von Bauhin geschaffene und benutzte Nomenklatur wird also gemäß einer sehr genauen Syntax strukturiert, in der die Reihenfolge der Konstituenten einer Benennung zur wesentlichen Definition einer Pflanzenform führt, indem sie das Verfahren der logischen Teilung bis zur äußersten Art wiederholt. Das letzte Epitheton (major in Valeriana sylvestris major) ist Träger der wesentlichen Differenz, die die Pflanze im Gegensatz $\mathrm{zu}$ den anderen Pflanzen ihrer Gattung wesensmäßig definiert. Einige Beispiele scheinen jedoch die Theorie der logischen Teilung zu widerlegen, besonders wenn man feststellt, dass eine Benennung wie z.B. (16) im Phytopinax im Pinax eine verkürzte Form (17) aufweist:

$$
\begin{aligned}
& \text { X. Valeriana peregrina caerulea. } \\
& \text { Blauer fremder Baldrian. } \\
& \text { Valeriana caerulea. } \\
& \text { Blauer Baldrian. }
\end{aligned}
$$

Diese Veränderung scheint die traditionelle Interpretation zu bestätigen, dass (17) ein Binom sei und keineswegs die logische Teilung wiederhole, dass (17) eher eine Art der höchsten Gattung Valeriana sei als eine Art der Zwischengattung Valeriana peregrina. Um die Interpretation der logischen Teilung zu vertreten, muss das Adjektiv peregrina mit darunter verstanden werden. Es ist durchaus möglich, eine solche Ellipse anzunehmen, da alle kurzen oder verkürzten Formen chronologisch nach den vollständigen Formen belegt sind. Zwei grundverschiedene Tatsachen, die den Grundgedanken der Episteme der Renaissance Rechnung tragen, bestätigen diese Annahme. 
Zuerst gibt es die Unbeständigkeit der typographischen Praxis. Im Hinblick darauf könnte man das Fehlen des Adjektivs peregrina als von Bauhin ungewollt betrachten. Während Bauhin alle Druckfehler der drei ersten Bücher seines Pinax verbessert hat, gibt es für die neun anderen keine absolute Zuverlässigkeit. Die Form (17), die zum vierten Buch gehört, könnte auf einen Druckfehler zurückzuführen sein, und in diesem Fall müsste peregrina wieder eingesetzt werden: Das ist gewiss eine schwache Hypothese, da die Fehlerquote bei den Namen der drei ersten Bücher etwa zwei Prozent beträgt. Für diese Hypothese spricht aber die Tatsache, dass Caspar Bauhin auf den handgeschriebenen Etiketten des Herbars nur die Form (16) benutzt.

Zweitens gibt es das Prinzip der kognitiven Ökonomie, das für die Ellipse des Adjektivs peregrina spricht. Während die Zahl der bekannten Pflanzen weiter zunimmt und die Pflanzennamen im Durchschnitt drei oder vier lexikalische Einheiten enthalten, weisen immer mehr Vorworte oder Bemerkungen in den Renaissancetexten auf die Notwendigkeit hin, das Auswendiglernen und Behalten der Pflanzennamen $\mathrm{zu}$ erleichtern. Diese Bemerkungen erinnern sehr an das von den modernen strukturalistischen Linguisten und Kognitivisten hervorgehobene Prinzip der kognitiven Ökonomie: die Wörter, besonders die zusammengesetzten Wörter, werden verkürzt, wenn sie häufig benutzt werden. Vielleicht liegt gerade dieses Prinzip der kognitiven Ökonomie dem häufigen Phänomen der nomenklatorischen Verkürzung zugrunde, die man vor allem auf den Etiketten des Herbars von Bauhin und in seinen letzten Druckwerken feststellt. So werden auf ein und demselben Blatt des Herbars die Exsikkaten einer weit verbreiteten Pflanze sowohl durch die Vollform (18), als auch durch die Kurzform (19) bezeichnet:

\author{
III. Valeriana sylvestris minor. \\ Kleinerer wilder Baldrian. \\ Valeriana minor. \\ Kleinerer Baldrian.
}

Die Kurzform ist nicht ambig, da sie in der Nähe der Vollform steht, und da sie die Differenz, die die Pflanze wesentlich definiert, d.h. das Epitheton minor, bewahrt. Die Ellipse kann auch zwei lexikalische Einheiten betreffen. Die sehr häufige Gattung (20) - sie entspricht der Valerianella olitoria (L.) Poll. in moderner Taxonomie - enthält eine Art, die im Herbar immer durch die verkürzte Form (21), und nicht durch die unbelegte vollständige Form (22) bezeichnet wird:

(20) Valeriana campestris inodora major. Größerer geruchloser Feld-Baldrian.

(21) Valeriana campestris abortiva. Steriler Feld-Baldrian.

(22) *Valeriana campestris inodora major abortiva.

*Steriler größerer geruchloser Feld-Baldrian. 
Wie die Form (19) enthält auch die Benennung (21) das Epitheton (abortiva), das die Pflanze wesentlich definiert. Es ist also möglich, dass dasselbe Prinzip der kognitiven Ökonomie im Fall der mit (17) benannten Pflanze funktioniert hat: (17) entspricht dem Polemonium caeruleum L. in der modernen Botanik und ist eine häufig vorkommende Pflanze, die alle Renaissancebotaniker in ihren Gärten anbauten - die Exsikkaten im Herbar von Bauhin stammen in der Tat aus seinem Basler Privatgarten. Die binomische verkürzte Form Valeriana caerulea könnte ein mnemonischer Vorteil sein, da sie nur das Epitheton (caerulea) bewahrt, das diesen Baldrian im Gegensatz zu allen anderen, die rosa, weiße oder rote Blüten haben, wesentlich definiert.

Welche Erklärung man auch vorzieht, auf jeden Fall kann man den Schluss ziehen, dass das Binom (wie im Fall der vorher analysierten Valeriana hortensis) keineswegs auf dem Linné'schen Modell beruht: Nur unter Berücksichtigung der zur Episteme des sechzehnten Jahrhunderts gehörigen Grundgedanken ist eine richtige Interpretation möglich ${ }^{5}$.

\section{Die prototypische Begriffsbildung, ein zweites strukturelles Prinzip}

Während die logische Teilung die innere Ordnung der Konstituenten einer Pflanzenbenennung erklärt, gilt es nun das Prinzip zu erläutern, das im Pinax die Reihenfolge und die Nummerierung der Benennungen der äußersten Arten innerhalb einer Gattung bestimmt (s. Abbildungen Nr. 1 und Nr. 3). Es soll auch gezeigt werden, auf welche Weise dieses Prinzip die Form der Benennungen beeinflusst.

\subsection{Die Reihenfolge der Benennungen}

Die Reihenfolge der Benennungen innerhalb der Gattung ist gar nicht klar erkennbar. Während in der Anfangszeit der Renaissance und in einem Werk wie dem Pinax Phyton von Gesner (Gesner 1542), die Pflanzenbenennungen einfach in alphabetischer Reihenfolge erscheinen, wird später diese Reihenfolge von einem ganz anderen Prinzip bestimmt, wie wir es schon in dem Phytopinax von 1596 beobachten können (siehe oben Beispiele 4-15). Es lässt sich folgende Reihenfolge feststellen: zuerst die kultivierten oder hortenses Pflanzen (4), dann die wilden oder sylvestres Pflanzen (5)-(6), unabhängig von dem Ort, an dem sie wachsen (7)-(10), und an letzter Stelle die ausländischen (peregrinae) oder exotischen Pflanzen (11)/(13); in jeder dieser Gattungen, zuerst die größeren oder majores Pflanzen (5)/(14), dann die kleineren oder minores (6)/(15); und schließlich in jeder dieser Gattungen oder innerhalb der Gesamtheit dieser Gattungen zuerst die Pflanzen, die einfarbig sind, eine ausgesprochene oder häufig vorkommende Farbe haben, dann die Pflanzen, deren Farbe gemischt, blass oder in der Gattung weniger häufig (13) ist, oder zuerst die riechenden und danach die 
geruchlosen Pflanzen (12)-(15). Wenn man andere Kapitel von Pflanzen im Phytopinax studiert, stellt man immer dieselbe Regelmäßigkeit in der Reihenfolge der Benennungen fest.

Diese Regelmäßigkeit beruht auf einer epistemischen Charakteristik der Renaissance, die ich $\mathrm{zu}$ zeigen versucht habe: die prototypische Begriffsbildung, im kognitiven und neoplatonischen Sinn des Terminus. Psychologen und Kognitivisten zufolge beruht die Struktur einer Kategorie nicht auf Individuen, die alle dieselben Eigenschaften haben, sondern auf einem typischen Vertreter, d.h. auf einem besten Exemplar, der als "Prototyp" aufgefasst wird; die anderen Fälle, die sich vom Prototyp unterscheiden, da sie weniger typische Vertreter der Kategorie sind, werden als "Randfälle" aufgefasst. Alle Vertreter einer Kategorie lassen sich also auf einem Kontinuum von typisch bis hin zu weniger typisch anordnen, das als “Typikalitätsgradient” bezeichnet wird (nach Rosch 1973, 1975; Lakoff 1987; Dubois 1991). Der kognitive Begriff des Prototyps stimmt in mehr als einer Hinsicht mit dem Prototyp im neoplatonischen Sinn überein, der die vollkommenste Verwirklichung einer idealen (abstrakten) Form, d.h. der beste Vertreter dieser Form ist, indem er die meisten Eigenschaften der Form hat.

In den Werken der Renaissance werden die Pflanzen als mehr oder weniger vollkommene Verwirklichungen der von Gott geschaffenen Formen oder Ideen von Pflanzen betrachtet. In dieser gemäß eines Typikalitätsgradienten strukturierten Erkenntnisweise wird diejenige Pflanze als erste betrachtet, die die ideale Form am vollkommensten verwirklicht: Größe, Einfarbigkeit, Geruch sind der Ausdruck einer vollkommenen Verwirklichung und machen die Pflanze zum Prototyp; an zweiter Stelle kommen die Pflanzen, die eine verminderte Verwirklichung der Qualitäten dieser Form (Kleinheit, gemischte oder verwaschene Farbe,...) aufweisen. Die in den Gärten kultivierten Pflanzen, die im Allgemeinen groß und einfarbig sind oder für die Gattung typische Farben haben, bilden echte Prototypen, während die wilden Pflanzen, kleiner und mit veränderlichen oder weniger typischen Farben Randfälle sind und an das Ende einer Gattung gestellt werden. Dies scheint die Erklärung der Reihenfolge der Gattungsbenennungen nicht nur im Phytopinax, sondern auch im Pinax zu sein. Es fällt im Pinax tatsächlich auf, dass sich die neuen Zwischengattungen Valeriana rubra und Valerianella am Ende der Gattung VALERIANA (be)finden. Aber diese Randstellung erklärt sich nach der Prototypentheorie durch die unvollkommenen morphologischen Charakteristiken der Pflanzen: die Gattung Valeriana rubra enthält Pflanzen mit roten Blüten, die als entfernt von den rosa oder weißen Blüten der prototypischen Baldriane aufgefasst werden; wie aus dem Verkleinerungssuffix - ella in Valerianella und aus den Synonymen der anderen Autoren, die das Epitheton minimum oder minus (s. Abbildung Nr. 1) enthalten, hervorgeht, umfasst die Gattung Valerianella Pflanzen, die alle sehr klein bzw. winzig sind, d.h. also unprototypische Pflanzen. 


\title{
3.2. Der Aufbau der Benennungen
}

Die beiden vorangehenden Beispiele zeigen, wie sich die Prototypikalität mit der logischen Teilung verbindet, um die Stellung einer Benennung im Verhältnis zu anderen zu bestimmen. Aber die Prototypikalität beeinflusst manchmal auch auf linguistischer Ebene die Struktur der Benennung. Dies lässt sich anhand von zwei Beispielen aus dem Pinax belegen.

\subsubsection{Die nomenklatorische Polysemie}

Das erste Beispiel einer nach dem Prinzip des Prototypischen aufgebauten nomenklatorischen Struktur findet sich in den zwei folgenden äußersten Arten (s. Abbildung Nr. 1, Pflanzen XVII und XIIX):

\author{
Valeriana rubra. \\ Roter Baldrian. \\ Valeriana rubra angustifolia. \\ Schmalblättriger roter Baldrian.
}

Aufgrund des schon eher (s. Abschnitt 2) durchgeführten linguistischen Vergleichs, ist zu schließen, dass es eine Zwischengattung Valeriana rubra gibt. Doch diese Zwischengattung trägt dieselbe Benennung wie die äußerste Art (23) - es ist mit anderen Worten bei der Benennung Valeriana rubra, die ebenso die Gattung als auch eine ihrer Arten bezeichnet, von echter Polysemie die Rede. Im oben untersuchten Fall der äußersten Art Valeriana hortensis liegt keine Polysemie vor, da es keine zweite Teilung gibt, und das Epitheton hortensis ist die wesentlich definierende Differenz. Da sich die Gattung Valeriana rubra aber in zwei Arten gliedert, würde man in (23) ein adjektivisches Epitheton erwarten, das die Art wesentlich definiert, so wie in (24) die Art durch das Epitheton angustifolia definiert wird. Das ist jedoch nicht der Fall.

Die Erklärung liegt in einer prototypischen Begriffsbildung, die die Ethnobotaniker seit langem in den Volksnomenklaturen entdeckt haben. Nehmen wir eine einzelne und isolierte Pflanze " $X$ " an; wenn eine menschliche Gemeinschaft viel später eine andere Pflanze "Y" entdeckt, die "X" ähnlich ist, erscheint die vorher bekannte Pflanze " $X$ " als Prototyp, während die chronologisch als zweite bekannt gewordene Pflanze "Y" als Randfall betrachtet wird. Dies führt dazu, dass, wenn aus den gemeinsamen Eigenschaften beider Pflanzen der Begriff einer hierarchisch höheren Gruppe abstrahiert wird, der Name dieser höheren Gruppe meistens aus dem Namen der prototypischen Pflanze, d.h. "X", entlehnt wird.

So ist die Pflanze (23) lange Zeit eine isolierte Pflanze geblieben, bevor sehr viel später die von (24) bezeichnete Pflanze entdeckt wurde - sie wurde erst im Prodromus von 1620 zum ersten Mal erwähnt. Infolgedessen erschien die früher bekannte und häufiger vorkommende Pflanze (23) als 
Prototyp, während (24) als atypischer Fall aufgefasst wurde. Der Name der Zwischengattung wurde dann aus dem Namen der prototypischen Art (23) entlehnt. Das bedeutet, dass einerseits die prototypische Begriffsbildung aufgrund eines ansteigenden Abstraktionsprozesses zur Benennung einer Zwischengattung, Valeriana rubra, führt; dass andererseits ausgehend von dieser Gattung die logische Teilung aufgrund eines absteigenden und additiven Prozesses zur Benennung einer äußersten Art, (24) Valeriana rubra angustifolia, führt. Doch die Benennung der prototypischen Art (23) wird, gerade wegen ihres Status eines Prototyps, von diesen beiden Prozessen nicht betroffen. Ein solcher polysemantischer Aufbau ist häufig in der Nomenklatur Bauhins und kann eine Zwischengattung der dritten Stufe betreffen. Auf diese Weise ist Valeriana sylvestris major eine polysemantische Benennung, die gleichzeitig die prototypische Art (25) und ihre Zwischengattung der dritten Stufe bezeichnet (s. Abbildung Nr. 3), während Valeriana sylvestris major montana eine monosemantische Benennung ist, die die andere untypische Art (26) derselben Zwischengattung bezeichnet und die ein die Pflanze wesentlich definierendes Epitheton (montana) enthält:
Valeriana sylvestris major.
Größerer wilder Baldrian.
Valeriana sylvestris major montana.
Bergiger größerer wilder Baldrian.

Kann man aus der Polysemie der Benennung Valeriana rubra folgern, dass die Nomenklatur Bauhins eine volkstümliche Nomenklatur ist? Die Ethnobotaniker (Berlin 1972; Atran 1987) sind der Meinung, dass eine solche Polysemie, die man in den volkstümlichen Kulturen der ganzen Welt beobachten kann, ein Beleg dafür sei. Die Polysemie neigt zwar dazu, die systematische Unterscheidung der polynomischen Zwischengattungen der zweiten (23) oder der dritten (25) Stufe zu verschleiern, so dass diese Zwischengattungen von den modernen Interpreten nicht als solche erkannt werden; diese Polysemie steht auch der systematischen Anwendung der logischen Teilung im Wege, da die Definition, die man bekommt, kein Epitheton, das die wesentliche Differenz ausdrückt, enthält. Da sie gar nicht systematisch ist, wird die Nomenklatur Bauhins als unwissenschaftlich betrachtet (s. Abschnitt 1.). Doch diese Beurteilung beruht auf der modernen Weltanschauung, die die Wissenschaft nur als System erfasst. Anstatt die modernen Kategorien Wissenschaftlichkeit und Volkstümlichkeit auf die Nomenklatur Bauhins anzuwenden, scheint es mir relevanter zu versuchen, den kognitiven Mechanismus dieser Struktur, die die logische Teilung mit der Prototypikalität verbindet, zu verstehen. 


\subsubsection{Die nomenklatorische Anapher}

Diesen kognitiven Mechanismus, der den Beispielen (23)-(24) zugrunde liegt, können wir anhand der folgenden Feststellung zeigen: das Wort angustifolia in (24) ist keiner einzigen linguistischen Form in (23) entgegengesetzt, aber das Fehlen eines definierenden Epithetons unterstreicht gerade den prototypischen Charakter von (23) im Vergleich zu (24). Das ist ein anaphorischer Mechanismus, in dem Sinne, dass die unmarkierte Form (23) nur in Zusammenhang mit der linguistisch markierten Form (24) ihre Bedeutung bekommt.

Solch ein Mechanismus zeigt sich noch deutlicher und mündet in eine ausdrücklichere anaphorische Beziehung, wenn sich die polysemantische Situation entwickelt. Ein Beispiel dieser Entwicklung findet sich in der Gattung NARDUS, die der Gattung VALERIANA folgt. Die Etiketten von Bauhins Herbar weisen die folgende ursprüngliche polysemantische Situation auf:

\section{Nardus Celtica.}

Keltisches Borstengras.

Nardus Celtica altera.

Keltisches Borstengras, das andere.

Hier bezeichnet Nardus Celtica sowohl die Zwischengattung als auch die prototypische äußerste Art (27), und dies ohne definierendes Epitheton, während die unprototypische Art (28) ein solches Epitheton (altera) bei sich hat ${ }^{6}$.

Diese Situation, wobei es zugleich eine den Prototyp (27) bezeichnende polysemantische Benennung gibt und das Adjektiv altera (28) zur Unterscheidung der untypischen Art, entwickelt sich im Allgemeinen in zwei Richtungen: die Benennung des Prototyps bekommt ein definierendes Attribut, das entweder (i) nicht anaphorisch oder (ii) anaphorisch in Form eines Zahlwortes ist:

(i) das nicht anaphorische Attribut ist oft der Name eines Autors der Antike: Die Werke von Theophrastus, Dioskorides und Plinius werden als Autoritäten für die Beschreibung der als prototypisch aufgefassten ersten Formen betrachtet. Deshalb verändert sich schon im Phytopinax (27) in (29):

Nardus Celtica Dioscoridis.

Keltisches Borstengras des Dioskorides.

In dieser Situation bleibt die anaphorische Beziehung einseitig: nur (28) ist anaphorisch mit (29) verbunden.

(ii) das anaphorische aus einem Zahlwort bestehende Attribut wird nur durch das Adjektiv erst ausgedrückt:

Alpen-Baldrian, der erste. 
Valeriana alpina altera.

Alpen-Baldrian, der andere.

Diesmal kommt eine doppelte Anapher zustande: (31) ist anders als (30) und (30) ist die erste von beiden. In dieser zwei Elemente umfassenden Namensbildung schlagen einige Latinisten vor, das Adjektiv altera mit zweite zu übersetzen. Diese Übersetzung erscheint mir als ungenau, da sie alter $(a, u m)$ zu einem Synonym von secundus ( $a$, um) macht:

Erstens verfügt Bauhin über das Zahlwort secundus. Er benutzt es in einer Aufzählung, in der eine Serie nummerierter Pflanzen (z.B. Anemone prima, Anemone secunda..., Anemone quinta, d.h. Erstes Windröschen, Zweites Windröschen ..., Fünftes Windröschen) benannt wird. In (31) aber verwendet er alter und dieses Adjektiv gilt es zu erklären;

Zweitens ergibt die Chronologie, dem Sinn des Adjektivs alter ('das andere von nur zwei') entsprechend, ganz deutlich, dass alter ursprünglich nicht in einer nummerierten Serie, d.h. in einer Aufzählung, erscheint, sondern dass es der unergänzten Benennung des Prototyps gegenübergestellt wird;

Drittens geht aus der Chronologie vor allem ganz deutlich hervor, dass das Adjektiv primus, das das Bedeutungsmerkmal ,Aufzählung ' enthält und das etwas ,zweites“ impliziert, erst später in der Benennung des Prototyps erscheint, im Augenblick, wo sich die Form alter schon in der Benennung der unprototypischen Pflanze findet. Das Adjektiv alter darf also nicht als Zahlwort aufgefasst werden: Die oben (s. Endnote 6) erläuterte Kontextbedeutung ,was nicht der Prototyp ist' trifft also eher zu. Ich würde das Paar alter/primus lieber mit anderen Paaren von Adjektiven wie adulterinus/verus oder minor/major vergleichen, die auf dieselbe Weise aufgebaut sind: Eine unprototypische Pflanze, die außerehelich (adulterinus) oder kleiner (minor) genannt wird, unterscheidet sich von einer prototypischen Pflanze, die a posteriori, durch Vergleich mit ihr, ehelich (verus) oder gröBer (major) genannt wird. Die Funktion des Adjektivs alter auf eine einfache willkürliche Nummerierung beschränken, heißt mit anderen Worten, die chronologische Reihenfolge des Erscheinens der Epitheta - zuerst alter, dann primus - zu vernachlässigen, und erschwert vor allem, durch das Weglassen der Chronologie, das Verstehen der prototypischen Begriffsbildung. Es ist für mich unwichtig, dass das Adjektiv alter im Allgemeinen als charakteristisch für das volkstümliche Funktionieren der Nomenklatur betrachtet wird: Meiner Meinung nach liegt auf kognitiver Ebene das Wichtigste in der Weise, wie die prototypische oder unprototypische Identität einer Pflanze durch das Fehlen eines Epithetons (27) bzw. das Vorhandensein eines Epithetons wie alter (28) linguistisch aufgebaut wird, und in der Art und Weise wie die anaphorische Beziehung ein Netz zwischen den Pflanzen knüpft und den Erwartungen der Botaniker entspricht, die die Beziehungen zwischen den Pflanzen untersuchen. 


\section{Schlussfolgerung}

In der Renaissance dienen die Pflanzenbenennungen drei verschiedenen Zwecken:

(i) Sie sollen die Pflanzennatur wesentlich definieren. Die Morphosyntax gibt diesen Zweck genauen Regeln gemäß wieder: die Anordnung der Konstituenten entspricht der Struktur einer logischen Definition, die einen Gattungsnamen mit einer Differenz verbindet, denn die Benennung fasst ein Verfahren der logischen Teilung zusammen; die Bedeutung der einzelnen Konstituenten wird durch ein prototypisches Prinzip bestimmt, das sich auf verschiedene Weisen verwirklicht, unter anderem durch die Polysemie (die typische Art trägt denselben Namen wie ihre Gattung) und die anaphorischen Beziehungen;

(ii) Sie sollen die Klassifizierung der Pflanzen repräsentieren, die ihrerseits die Beziehungen zwischen den von Gott geschaffenen Pflanzen wiedergeben soll. Die Reihenfolge der Benennungen entspricht also genauen Regeln: die Benennungen werden gemäß eines Typikalitätsgradienten, vom Prototyp bis hin zu den Atypischen angeordnet;

(iii) Sie sollen die Pflanzen draußen in der Natur praktisch bezeichnen, ohne das Gedächtnis übermäßig zu belasten, was dazu führt, dass die logischen Definitionen auf einfache Binome reduziert werden.

Aus den oben erklärten Zwecke können wir schließen, dass es sich tatsächlich um eine Nomenklatur, d.h. eine Gesamtheit nach bestimmter Prinzipien strukturierter Benennungen, handelt, die der Episteme der Renaissance eigen sind.

\section{Bibliographie}

Aristoteles (1991). Métaphysique (Ed. J. Tricot). 2 Bände. Paris: Vrin.

Aristoteles (1997). Les Topiques (Ed. J. Tricot). Paris: Vrin.

Atran, Scott Atran (1987). "Origin of the Species and Genus Concepts: An Anthropological Perspective." Journal of the History of Biology 20(2), 195-279.

Bauhin, Caspar (1596). Phytopinax seu Enumeratio plantarum. Basileae: Petri.

Bauhin, Caspar (1623). Pinax Theatri Botanici. Basileae: König.

Bauhin, Caspar (s.d.). Etiketten des Herbars. Basler Botanisches Institut.

Berlin, Brent (1972). "Speculation on the growth of botanical nomenclature." Language and Society 1, 51-86.

Cain, Arthur James (1994). "Rank and sequences in Caspar Bauhin's Pinax." Botanical Journal of the Linnean Society 114, 311-356.

Dubois, Danièle (ed.) (1991). Sémantique et cognition. Catégories, prototypes, typicalité. Paris: CNRS.

Foucault, Michel (1966). Les mots et les choses. Paris: Gallimard.

Freedman, Joseph (1993). "Aristotle and the Content of Philosophy Instruction at Central European Schools and Universities during the Reformation Era (1500-1650)." Proceedings of the American Philosophical Society 137(2), 213-253. 
Gesner, Conrad (1542). Catalogus Plantarum. Tiguri: Froschouerus.

Hünemörder, Christian (1983). "Aims and intentions of botanical and zoological classification in the middle ages and Renaissance." Pubblicazioni della stazione zoologica di Napoli, History and Philosophy of the life sciences II,5(1), 53-67.

Lakoff, George (1987). Women, fire and dangerous things. Chicago: The University of Chicago Press.

Möbius, Martin (1937). Geschichte der Botanik. Jena: Fischer.

Pajaud, Daniel (1989). La taxinomie bionaturaliste. Paris: Lachurié.

Porphyrus (1998). Isagoge (Ed. A. de Libera). Paris: Vrin.

Rosch, Eleanor (1973). "Natural Categories." Cognitive Psychology 4, 328-350.

Rosch, Eleanor (1975). "Cognitive presentation of Semantic Categories." The Journal of Experimental Psychology 104, 192-233. ${ }^{1}$ Im allgemeinen Sprachgebrauch bestehen die Namen aus einem einzigen Wort, aber
in der Renaissance bestehen sie oft aus mehr als einem Wort. Darum verwende ich
den Terminus Benennung als Synonym für Namen, um die Pflanzennamen aus der
Zeit vor Linné zu bezeichnen, ungeachtet der Anzahl der Wörter. Die Nomenklatur
ist die Gesamtheit der Benennungen eines Gebiets. Ich benutze auch den Terminus
Bezeichnung, entweder um das Bezeichnen, oder um das Ergebnis des Bezeichnens,
d.h. einen kurzen Namen, der wie eine Etikette auf einen Gegenstand hinweist, zu
bezeichnen; im Allgemeinen ist bezeichnen in diesem Artikel ein Synonym für auf
etwas hinweisen.

${ }^{2}$ Mit Episteme - einem Terminus, den ich von Foucault (Foucault 1966) übernehme - meine ich die zu einer bestimmten Epoche gehörenden anonymen und unbewussten Ordnungsstrukturen des Wissens, die die Wissenschaften prägen; sie ist also der Rahmen innerhalb dessen positives Wissen gewonnen werden kann.

${ }^{3}$ Die vom binomischen Linné'schen Modell beherrschte Tradition (Hünemörder 1983) schränkt den Gattungsnamen auf eine einzige lexikalische Einheit ein: In den vielen Fällen, in denen wie in (5) der Gattungsname mehr als eine lexikalische Einheit umfasst, läuft eine solche Analyse darauf hinaus, dass einerseits das Vorhandensein von zwei Teilungsstufen, andererseits die Begriffsbildung einer eigenen Gattung (Valeriana sylvestris) verborgen wird; und in einem Fall wie (4) Valeriana hortensis, der aus einer einzigen Teilungsstufe hervorgeht, verhüllt diese traditionelle Linné'sche Analyse, wenn sie gültig erscheinen mag, eigentlich die logische und formale Begriffsbildung der Beziehungen zwischen Art und Gattung.

${ }^{4}$ Ein Historiker (Cain 1994) hat auch den logischen Gebrauch der Termini Art und Gattung in Bauhins Pinax hervorgehoben. Er hat jedoch weder die das Pflanzenwesen definierende logische Struktur der Nomenklatur noch die Existenz verschiedener Gattungstufen erklärt.

5 Zum Schluss möchte ich auch die Veränderlichkeit der nomenklatorischen Verwirklichungen unterstreichen: In diesem Kontext soll daran erinnert werden, dass es sich in der Renaissance immer nur um Tendenzen oder allgemeine Prinzipien handelt, deren Anwendung nicht systematisch ist und deren Verbindung zu keinem lesbaren oder homogenen Ergebnis führt. Einer Episteme, die noch keine Abgrenzung der Disziplinen kennt und nicht zwischen volkstümlich und wissenschaftlich unterscheidet, ist der Systematismus völlig fremd.

${ }^{6}$ Die Differenz altera, die (28) wesentlich definiert, kann einen aktuellen Leser sehr überraschen, denn es bezeichnet keine positive und objektive Eigenschaft der Pflanze, sondern ihre Beziehung zu einer anderen Pflanze vom subjektiven Gesichtspunkt des Beobachters aus. Zunächst ist es in der Episteme der Renaissance 
nicht außergewöhnlich, dass die Eigenschaft häufiger der Kenntnis, die die Beobachter von der Pflanze haben, als der eigentlichen Morphologie der Pflanze entnommen wird. Die Schöpfung der Pflanzen wird tatsächlich immer im Zusammenhang mit dem Menschen betrachtet: die Pflanzen sind dem Menschen nützlich, entweder als Heilpflanzen, die den menschlichen Körper heilen, oder als Futterpflanzen, die das vom Menschen gezüchtete Vieh ernähren, oder als ästhetische Pflanzen, die das menschliche Auge erfreuen, oder als Gottesschöpfung, die den Menschen an die Existenz des Schöpfers erinnert. Da außerdem das Adjektiv alter $(a, u m)$, das andere von nur zwei' bedeutet, meint man, wenn man sagt, dass eine bestimmte Pflanze die andere (altera) ist,

- was sie ist: die Pflanze, die weniger häufig als der Prototyp vorkommt und im Allgemeinen später als dieser Prototyp entdeckt worden ist - wie auch im hiesigen Fall;

- was sie nicht ist: der Prototyp, d.h. die Pflanze, die wegen ihrer Häufigkeit und ihrer früheren Entdeckung als die vollkommene und prototypische Verwirklichung einer Pflanzenform betrachtet wird.

Dies trägt also dazu bei, sie in das Beziehungsnetz der Pflanzenformen, das die Botaniker ans Licht zu bringen versuchen, unter dem Gesichtspunkt des Wesens einzuordnen: Diese Pflanze wird also immer gleichzeitig durch ihre Nähe zum Prototyp (sie folgt ihm in der Klassifizierung) und durch ihre Verschiedenheit (im Unterschied zum Prototyp ist sie im Allgemeinen weniger groß und ihre Farbe ist weniger ausgesprochen) erfasst. 

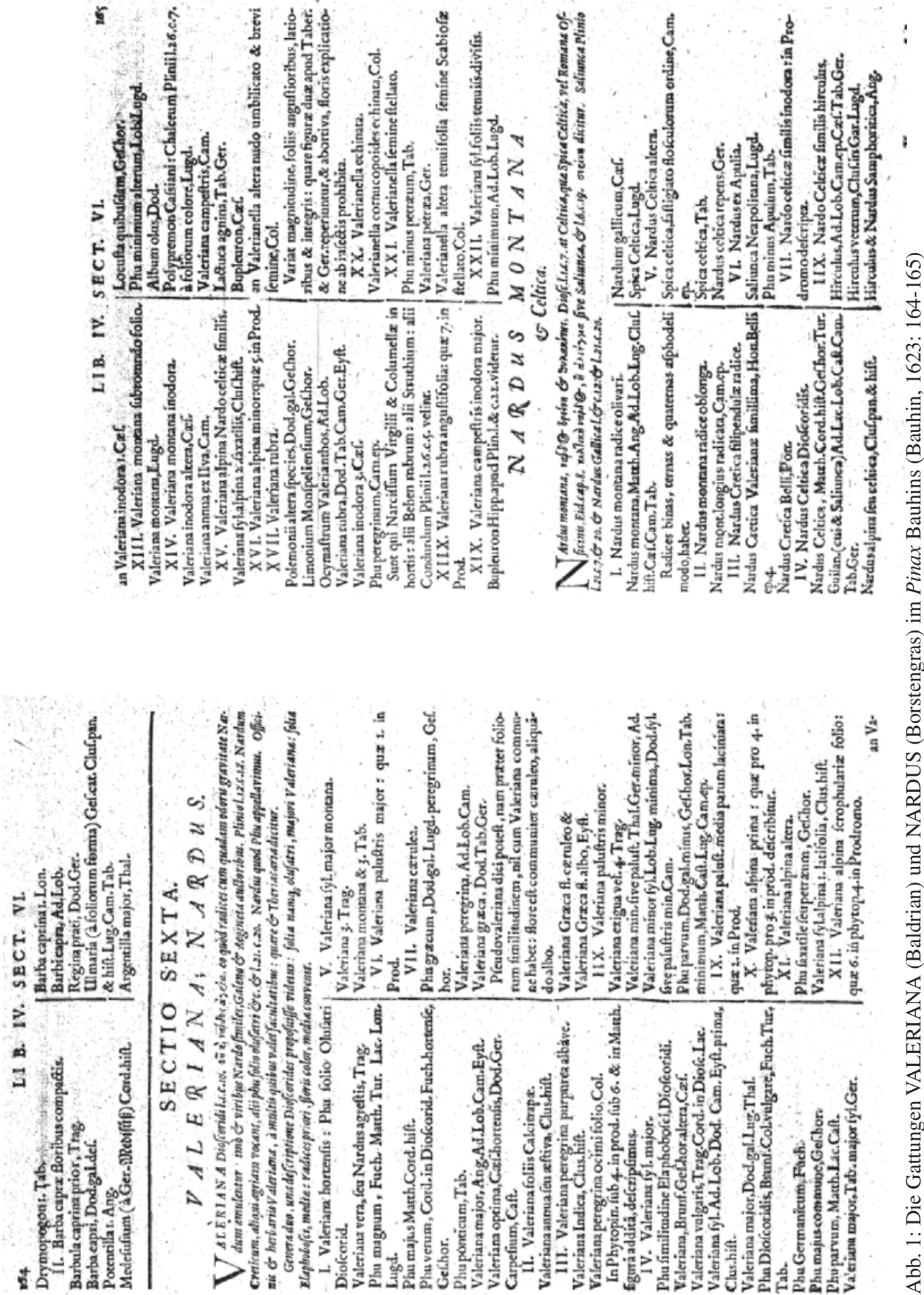


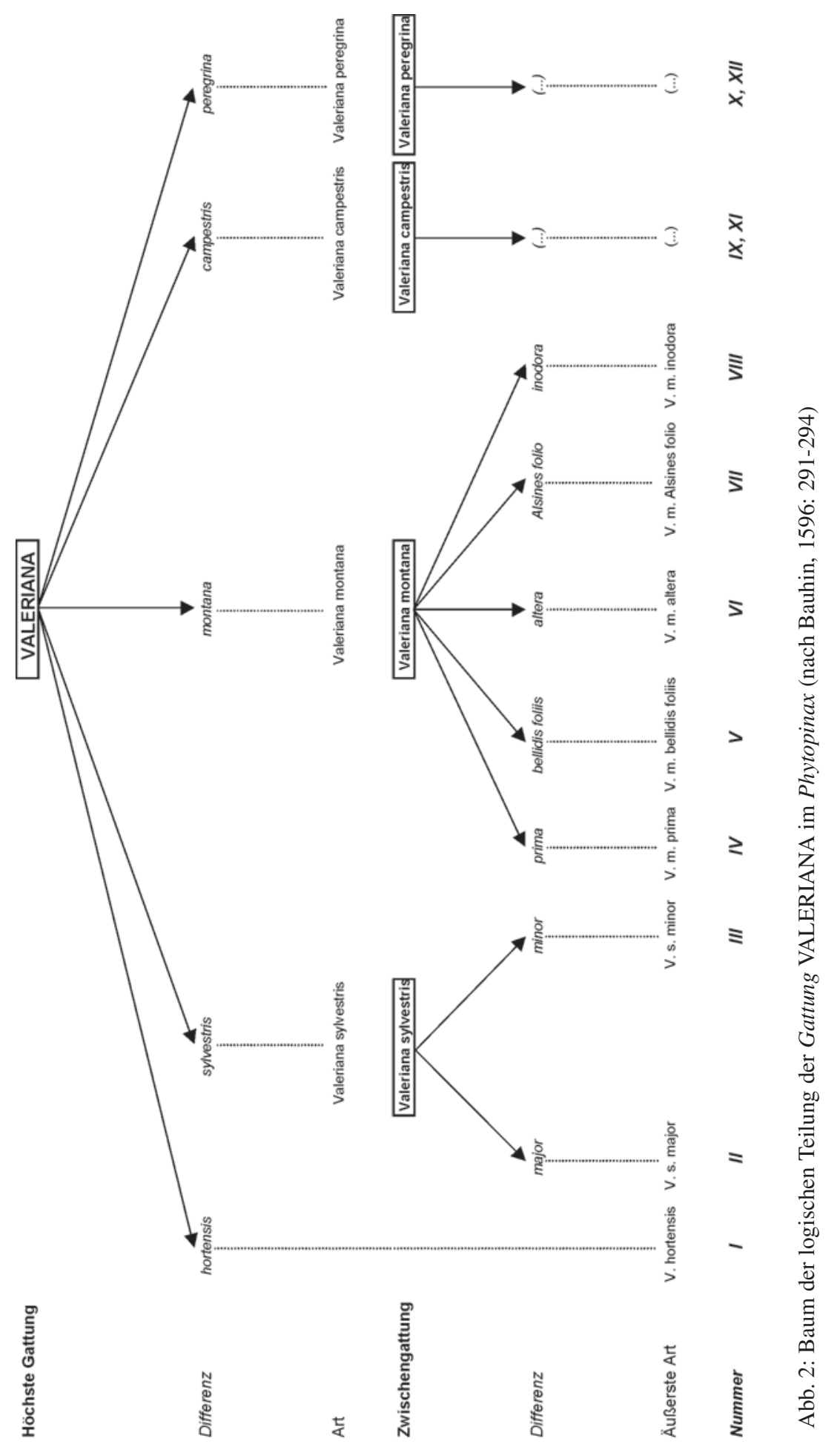




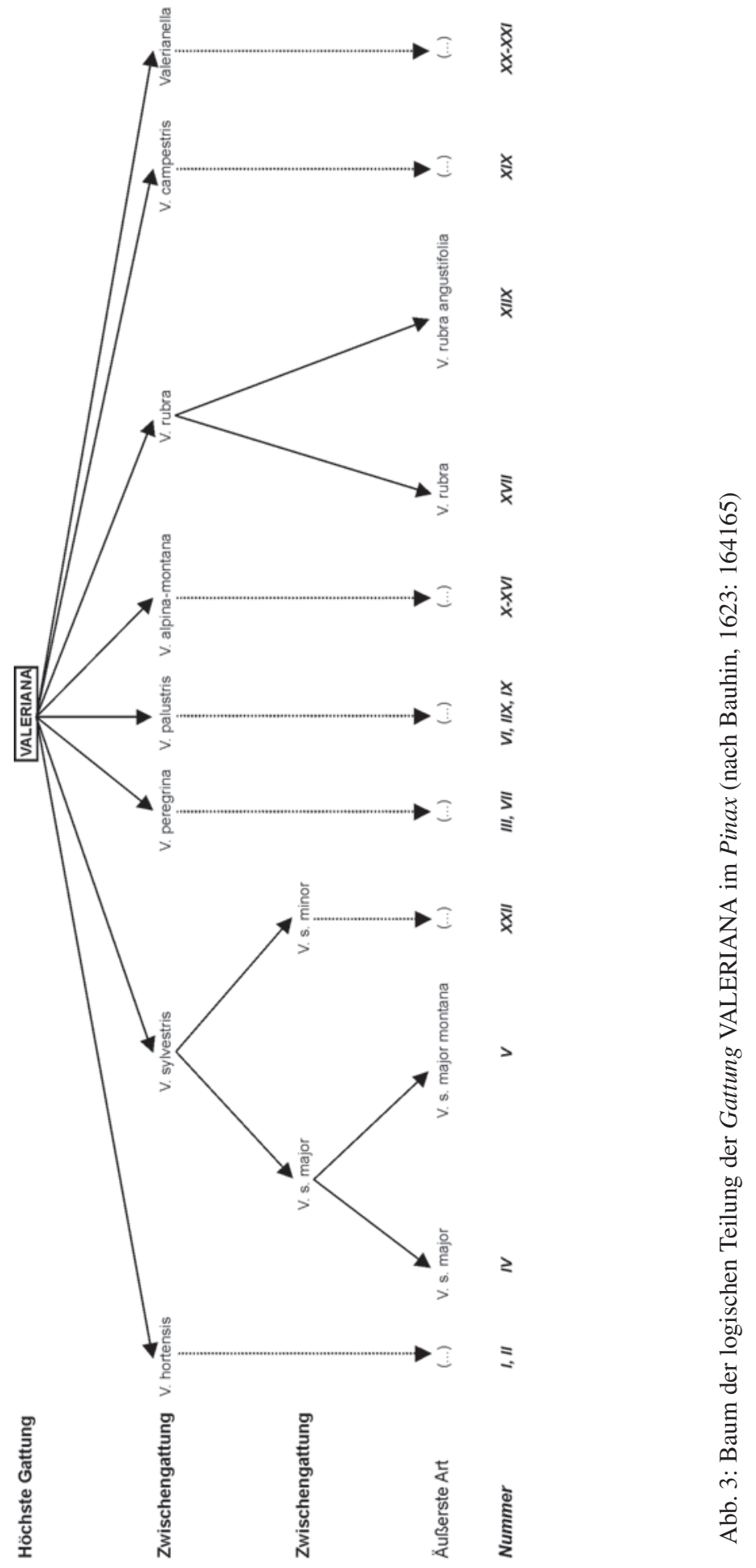




\section{$L A \mathcal{P} A \mathcal{T} H V M E T E I V S$ \\ Species.}

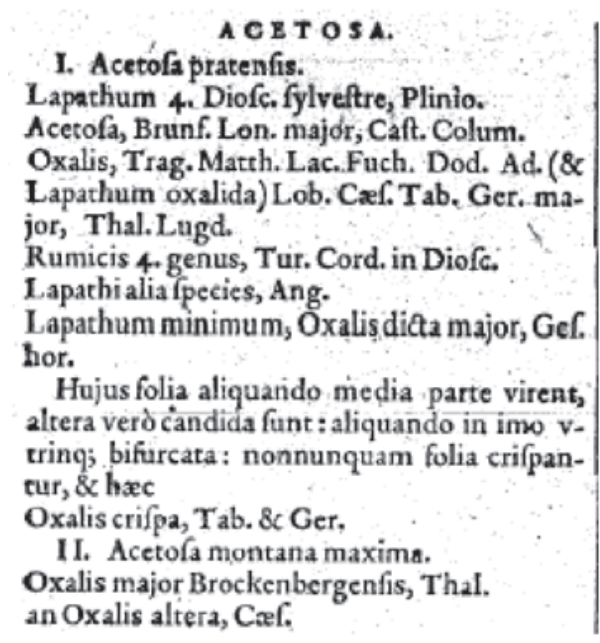

\author{
L A RATH T \\ Lapathwm horten/e.
}

I. Lapathum hortenfe folio oblongo, five fecundum Diofcoridis.

Rumex hort. vel 2. Trag, fativus, Cord, in Diofc.

Lapathum hortenfe, Gef. hort. Lugd.

Lapathum fativum, Ang. Dod. defcr.

Hippolapathum fyl. Matth. Lugd.

II. Lapathum hort: fcu Spinacia femine fpinofo.

Spinachia, Brunf.Fuch. Dod. Tab.

Spinacia, Matth. Ad. Lob. Lugd. Cxf. Ger. mas, $\mathrm{C}$ am.

Spanachia, Matth. Lon. Caft.

Olus Hifpanicum Spinachia vulgaris, Trag.

Spinaceum olus, Ger. hor.

Spinachia, ob fermina dür \& f pinofa, Grecis

Abb. 4: Die typographische Neuheit des Pinax (Bauhin, 1623: 113-114)

- in Großbuchstaben: die höchste Gattung (LAPATHUM);

- in kleineren Großbuchstaben: die Zwischengattungen der zweiten Stufe (ACETOSE, LAPATHUM);

- in kursiven Kleinbuchstaben: die Zwischengattung der dritten Stufe (Lapathum hortense);

- in römischen Buchstaben: die äusersten Arten (I. Acetosa pratensis, I. Lapathum hortense folio oblongo ...).

N.B.: Das Funktionieren einer Benennung wie Lapathum, die gleichzeitig die höchste Gattung und die Zwischengattung bezeichnet, wird im Abschnitt 3.2.1. erklärt. 